\title{
A Note on Fuzzy Random Vectors as Models for Vague but Synthetic Descriptions of Complex Random Phenomena
}

\author{
Tokuo Fukuda
}

\author{
Faculty of Management, Otemon Gakuin University, \\ 2-1-15 Nishi-Ai, Ibaraki, Osaka, 567-8502, JAPAN \\ Phone: Int.+81(Japan)-726-41-9704 \\ Fax: Int.+81(Japan)-726-43-9432 \\ E-Mail: fukuda@res.otemon.ac.jp
}

\begin{abstract}
In this paper, the author will investigate a class of fuzzy random vectors, where FRVCs are considered as vague but smart perceptions of some complex random phenomena.

First, based on the result previously proposed by the author[1], and inspired by the recent researches, especially Krätschmer[2], the definition of FRVCs are reconsidered form the viewpoint of vague but concise description of the state of very complex random phenomena.

Secondly, the expectation of FRVCs are derived from the viewpoint of mult-valued logic approach.
\end{abstract}

\section{Topology in Compact Convex Subsets of $\mathbb{R}^{n}$}

Let $\mathbb{R}^{n}$ be the $n$-dimensional Euclidean space. Then, it is well-known that $\mathbb{R}^{n}$ is a real separable Banach space(complete metric space) with the norm $\|x\|=$ $\left[\sum_{i=1}^{n} x_{i}^{2}\right]^{\frac{1}{2}}$ for any point $x=\left(x_{1}, x_{2}, \cdots, x_{n}\right)^{\prime}$ of $\mathbb{R}^{n}$. Let $\mathrm{K}_{\mathrm{cc}}\left(\mathbb{R}^{n}\right)$ denote the family of all nonempty compact convex subset of $\mathbb{R}^{n}$. Then, (Minkowski)addition and scalar multiplication between two elements of $A$ and $B$ are defined by

$$
\begin{aligned}
A+B & =\{a+b: a \in A, b \in B\} \\
\lambda A & =\{\lambda a: a \in A\},
\end{aligned}
$$

and they are closed in $\mathrm{K}_{\mathrm{cc}}\left(\mathbb{R}^{n}\right)$. The Hausdorff distance between the sets $A$ and $B$ is given by

$$
d_{H}(A, B)=\max \left\{\sup _{a \in A} \inf _{b \in B}\|a-b\|, \sup _{b \in B} \inf _{a \in A}\|a-b\|\right\},
$$

where $\|\cdot\|$ is the Euclidean norm. Then, it is well known that the Hausdorff distance is a metric on $\mathrm{K}_{\mathrm{cc}}\left(\mathbb{R}^{n}\right)$. The magnitude of $A$ is defined by

$$
\|A\|_{H}=d_{H}(A,\{0\})=\sup _{a \in A}\|a\| .
$$

Let $\tau_{d_{H}}$ be the topology on $\mathrm{K}_{\mathrm{cc}}\left(\mathbb{R}^{n}\right)$ induced by the Hausdorff metric. Then, it is known that $\left(\mathrm{K}_{\mathrm{cc}}\left(\mathbb{R}^{n}\right), \tau_{d_{H}}\right)$ is a locally compact, $\sigma$-compact Polish space, and the product space $\left(\mathrm{K}_{\mathrm{cc}}\left(\mathbb{R}^{n}\right)\right)^{(0,1] \cap \mathbb{Q}}$ endowed with product topology $\tau_{\Pi d_{H}}$ with respect to $\tau_{d_{H}}$ is a Polish space[2].

\section{Fuzzy Logic as Multi-valued Logic}

First, according to Kwakernaak's paper[3], we give a brief discussion of fuzzy logic as a special multi-valued logic. To this end, consider a complete distributive lattice of vague statements. The lattice is defined by a set of vague statements $\mathcal{P}$ and the binary operations $\wedge$ ("and") and $\vee$ ("or"). The connectives possess the usual properties of

$$
\begin{aligned}
& \text { idempotency } a \vee a=a \text { and } a \wedge a=a \text { for arbi- } \\
& \text { trary } a \in \mathcal{P}, \\
& \text { commutativity } a \vee b=b \vee a \text { and } a \wedge b=b \wedge a \\
& \text { for arbitrary } a, b \in \mathcal{P}, \\
& \text { associativity }(a \vee b) \vee c=a \vee(b \vee c) \text { and }(a \wedge \\
& b) \wedge c=a \wedge(b \wedge c) \text { for arbitrary } a, b, c \in \mathcal{P},
\end{aligned}
$$

absorption identity $a \vee(a \wedge b)=a$ and $a \wedge(a \vee$ $b)=a$ for arbitrary $a, b \in \mathcal{P}$.

Distributivity

$$
a \vee(b \wedge c)=(a \vee b) \wedge(a \vee c)
$$

and

$$
a \wedge(b \vee c)=(a \wedge b) \vee(a \wedge c)
$$

is also assumed, and moreover the lattice is supposed to be complete, which means that statements such as

$$
\bigvee_{\lambda \in \Lambda} a_{\lambda}, \bigwedge_{\lambda \in \Lambda} a_{\lambda}
$$

with $a_{\lambda} \in \mathcal{P}$ for each $\lambda \in \Lambda$, are well defined and contained in $\mathcal{P}$. Here $a_{\lambda}, \lambda \in \Lambda$, is an indexed subset of statements.

On $\mathcal{P}$ we define a function $t: \mathcal{P} \rightarrow[0,1]$, where for given $a \in \mathcal{P}$, the number $t(a)$ is referred to as the "truth value" of the statement $a$. In usual mathematical logic, $t(a)$ assumes one of the two values 0 ("false") or 1 ("true"). In multi-valued logic, of which we are considering a particular instance, $t(a)$ may assume intermediate values. Bellman and Giertz[4] impose the following requirements on the function $t$ :

(a) There exist functions $f$ and $g$, both mapping $[0,1] \times$ $[0,1]$ into $[0,1]$, such that

$$
t(a \wedge b)=f(t(a), t(b)), \quad t(a \vee b)=g(t(a), t(b))
$$

for all $a \in \mathcal{P}$ and $b \in \mathcal{P}$. 
(b) $f(x, y)$ and $g(x, y)$ are continuous and nondecreasing in $x$.

(c) $f(x, x)$ and $g(x, x)$ are strictly increasing in $x$.

(d) $f(x, y) \leq \min (x, y)$ and $g(x, y) \geq \max (x, y)$ for all $x \in$ $[0,1]$ and $y \in[0,1]$.

(e) $f(1,1)=1$ and $g(0,0)=0$.

Property (a) expresses that the truth value of a compound statement is uniquely determined by the truth values of the component statements; this is called strict truth functionality in multi-valued logic. Property (b) expresses that $t(a \wedge b)$ and $t(a \vee b)$ do not become less true if $a$ is changed so that its truth value increases, and that moreover the dependence is continuous. Property (c) is equally plausible. Property (d) requires that the truth value of the statement $a \wedge b$ cannot be greater than the individual truth values of $a$ and $b$; similarly, the truth value of the statement $a \vee b$ is required to be at least as large as the individual truth values of $a$ and $b$. Finally, property (e) expresses that if $a$ and $b$ are both completely true, then also $a \wedge b$ is completely true, while if $a$ and $b$ are both completely false, then $a \vee b$ is also completely false.

Bellman and Giertz[4] have proved that (a) through (e) imply that the functions $f$ and $g$ are uniquely, given by

$$
f(x, y)=\min (x, y), \quad g(x, y)=\max (x, y) .
$$

We shall refer to the corresponding multi-valued logic as fuzzy logic. It is the logic introduced by Zadeh in his work on fuzzy sets. We observe that if the truth function is restricted to assume the values 0 and 1 only, we obtain the usual Boolean logic.

The interval $[0,1]$ together with the min and max operations forms another complete distributive lattice. Hence, $t$ is a homomorphism. It follows that for any subset of statements $a_{\lambda} \in P, \lambda \in \Lambda$, with $\Lambda$ an index set,

$$
t\left(\bigwedge_{\lambda \in \Lambda} a_{\lambda}\right)=\inf _{\lambda \in \Lambda} t\left(a_{\lambda}\right), \quad t\left(\bigvee_{\lambda \in \Lambda} a_{\lambda}\right)=\sup _{\lambda \in \Lambda} t\left(a_{\lambda}\right) \text {. }
$$

\section{Set Representation of Fuzzy Sets}

In this paper, a fuzzy set $\widetilde{U}$ given as a vague perception of $u_{0} \in \mathbb{R}^{n}$ is defined by the triple

$$
\widetilde{U}=\left(\mathbb{R}^{n},\left\{\widetilde{U}_{\alpha} \mid \alpha \in I\right\}, s_{\widetilde{U}}\right),
$$

where $\mathbb{R}^{n}$ is the $n$-dimensional Euclidean space called the basic space; $s_{\widetilde{U}}$ is the predicate, i.e., $s_{\widetilde{U}}: \mathbb{R}^{n} \rightarrow \mathcal{P}$ with $\mathcal{P}$ the "universe of discourse" defined by a set of statements, assigns a proposition

$$
s_{\widetilde{U}}(x)=\left\{x \text { coincides with } u_{\mathrm{o}}\right\}
$$

to each element $x \in \mathbb{R}^{n}[5]$; and $\left\{\widetilde{U}_{\alpha} \mid \alpha \in I\right\}$ with $I=[0,1]$ is the family of subsets of $\mathbb{R}^{n}$ satisfying

$$
L_{\alpha} \widetilde{U} \subseteq \widetilde{U}_{\alpha} \subseteq L_{\bar{\alpha}} \widetilde{U} \quad \text { for } \forall \alpha \in(0,1)
$$

and

$$
\widetilde{U}_{1}=\bigcap_{\alpha \in(0,1) \cap \mathbb{Q}} \widetilde{U}_{\alpha}, \quad \widetilde{U}_{0}=\mathrm{cl} . \bigcup_{\alpha \in(0,1) \cap \mathbb{Q}} \widetilde{U}_{\alpha} .
$$

where $\mathbb{Q}$ is the set of all rational numbers. In (8a) and (8b), $L_{\alpha} \widetilde{U}$ and $L_{\widetilde{\alpha}} \widetilde{U}$ are the strong cut and the level set of $\widetilde{U}$ at the level $\alpha$ defined respectively by

$$
\begin{array}{ll}
L_{\alpha} \widetilde{U}=\left\{x \mid\left(x \in \mathbb{R}^{n}\right) \wedge((\widetilde{U})(x)>\alpha)\right\} & \text { for } \alpha \in[0,1) \\
L_{\bar{\alpha}} \widetilde{U}=\left\{x \mid\left(x \in \mathbb{R}^{n}\right) \wedge((\widetilde{U})(x) \geq \alpha)\right\} & \text { for } \alpha \in(0,1],
\end{array}
$$

where $(\widetilde{U})(x)$ is the membership function of $\widetilde{U}$ given by

$$
(\widetilde{U})(x)=t\left(s_{\widetilde{U}}(x)\right)
$$

and $t(*)$ in (10) is the truth function of $*$ in the sense of multi-valued logic given in Sec. 2. The crisp point $u_{\mathrm{o}}$ in $\mathbb{R}^{n}$, the vague perception of which gives the fuzzy set $\widetilde{U}$, is called the original point of $\widetilde{U}$. The definition of fuzzy sets adopted in this paper is a modified one originally proposed by Kwakernaak[3]. Furthermore, we call the family of subsets $\left\{\widetilde{U}_{\alpha} \mid \alpha \in I\right\}$ defined by (8a) and (8b) the set representation of a fuzzy set $\widetilde{U}$. This view is slightly but not essentially different from its usual definition(see e.g. Kruse [6, 7]).

The set representation of the fuzzy set under discussion is assumed to satisfy the following conditions: (i) $\widetilde{U}_{1} \neq \emptyset$, (ii) $\widetilde{U}_{\alpha} \in \mathrm{K}_{\mathrm{cc}}\left(\mathbb{R}^{n}\right)$ for each $\alpha \in I$, (iii) $u_{\mathrm{o}} \in \widetilde{U}_{0}$, and the family of those satisfying (i), (ii) and (iii) is denoted by $\mathrm{F}_{\mathrm{cc}}\left(\mathbb{R}^{n}\right)$.

Let $\widetilde{U}=\left(\mathbb{R}^{n},\left\{\widetilde{U}_{\alpha} \mid \alpha \in(0,1)\right\}, s_{\widetilde{U}}\right)$ be an arbitrary fuzzy set. Then, it can be shown that

$$
\begin{aligned}
& L_{\alpha} \widetilde{U}=\bigcup_{\beta \in(\alpha, 1) \cap \mathbb{Q}} \widetilde{U}_{\beta} \text { for } \alpha \in[0,1), \\
& L_{\bar{\alpha}} \widetilde{U}=\bigcap_{\beta \in(0, \alpha) \cap \mathbb{Q}} \widetilde{U}_{\beta} \text { for } \alpha \in(0,1],
\end{aligned}
$$

and it also can be shown that $L_{\bar{\alpha}} \widetilde{U}$ is compact for each $\alpha \in I$ if $\widetilde{U} \in \mathrm{F}_{\mathrm{cp}}\left(\mathbb{R}^{n}\right)$. Furthermore, if $\widetilde{U} \in \mathrm{F}_{\mathrm{cc}}\left(\mathbb{R}^{n}\right), L_{\bar{\alpha}} \widetilde{U}, L_{\alpha} \widetilde{U}$ are also convex for each $\alpha \in I$.

As seen in (11a) and (11b), there are many fuzzy sets $\tilde{U}$ and $\widetilde{V}$, which have same strong cuts and level sets for each level $\alpha \in I$, and this relation is an equivalence relation $\sim$. Hence, we define the equivalence classes of fuzzy sets by $\mathbb{F}_{\mathrm{cp}}\left(\mathbb{R}^{n}\right)=\mathrm{F}_{\mathrm{cp}}\left(\mathbb{R}^{n}\right) / \sim$ and $\mathbb{F}_{\mathrm{cc}}\left(\mathbb{R}^{n}\right)=\mathrm{F}_{\mathrm{cc}}\left(\mathbb{R}^{n}\right) / \sim$.

Since $\mathbb{Q}$ is dense in $\mathbb{R}$, every fuzzy set is uniquely determined by its positive rational level sets. In particular, the mapping

$$
\begin{aligned}
& \pi: \mathbb{F}_{\mathrm{cc}}\left(\mathbb{R}^{n}\right) \rightarrow\left(\mathrm{K}_{\mathrm{cc}}\left(\mathbb{R}^{n}\right)\right)^{(0,1] \cap \mathbb{Q},} \\
& \widetilde{U} \mapsto\left(\bigcap_{\beta \in(0, \alpha) \cap \mathbb{Q}} \widetilde{U}_{\beta}\right)_{\alpha \in(0,1] \cap \mathbb{Q}}
\end{aligned}
$$

is injective. Then, the product topology $\tau_{\Pi d_{H}}$ with respect to $\tau_{d_{H}}$ will be translated to $\mathbb{F}_{\mathrm{cc}}\left(\mathbb{R}^{n}\right)$. 
Definition 3.1. A product topology on $\mathbb{F}_{\mathrm{cc}}\left(\mathbb{R}^{n}\right)$ is defined by

$$
\tau_{\mathbb{F}_{\mathrm{cc}}}=\left\{\pi^{-1}(G) \mid G \in \tau_{\Pi d_{H}}\right\}
$$

Let $d$ be a complete metric on $\left(\mathrm{K}_{\mathrm{cp}}\left(\mathbb{R}^{n}\right)\right)^{(0,1] \cap \mathbb{Q}}$ which is compatible with $\tau_{\Pi d_{H}}$. The induced mapping $d_{\mathbb{F}_{\mathrm{cc}}}=$ $d \circ(\pi, \pi)$ defines a metric on $\mathbb{F}_{\mathrm{cc}}\left(\mathbb{R}^{n}\right)$ since $\pi$ is injective, and $d_{\mathbb{F}_{\mathrm{cc}}}$ is compatible with $\tau_{\mathbb{F}_{\mathrm{cc}}}$. Then, since $\left(\left(\mathrm{K}_{\mathrm{cp}}\left(\mathbb{R}^{n}\right)\right)^{(0,1] \cap \mathbb{Q}}, \tau_{\Pi d_{H}}\right)$ is a Polish space, $\left(\mathbb{F}_{\mathrm{cc}}\left(\mathbb{R}^{n}\right), \tau_{\mathbb{F}_{\mathrm{cc}}}\right)$ is separable metrizable topological space.

Since $\left(\mathbb{F}_{\mathrm{cc}}\left(\mathbb{R}^{n}\right), \tau_{\mathbb{F}_{\mathrm{cc}}}\right)$ is homeomorphic to the topological subspace $\pi\left(\mathbb{F}_{\mathrm{cc}}\left(\mathbb{R}^{n}\right)\right)$ of $\left(\left(\mathrm{K}_{\mathrm{cp}}\left(\mathbb{R}^{n}\right)\right)^{(0,1] \cap \mathbb{Q}}, \tau_{\Pi d_{H}}\right)$, we can embed $\mathbb{F}_{\mathrm{cc}}\left(\mathbb{R}^{n}\right)$ into Polish space $\left(\left(\mathrm{K}_{\mathrm{cp}}\left(\mathbb{R}^{n}\right)\right)^{(0,1] \cap \mathbb{Q}}, \tau_{\Pi d_{H}}\right)$ as a Borel subset. Moreover, every Borel subset of $\mathbb{F}_{\mathrm{cc}}\left(\mathbb{R}^{n}\right)$ can be regarded as a Borel subset of $\left(\mathrm{K}_{\mathrm{cp}}\left(\mathbb{R}^{n}\right)\right)^{(0,1] \cap \mathbb{Q}}$. Then, it can be shown that [2]

$$
\pi\left(\mathbb{F}_{\mathrm{cc}}\left(\mathbb{R}^{n}\right)\right) \in \mathcal{B}_{\Pi d_{H}}\left(\mathbb{R}^{n}\right)
$$

For every $B \in \mathcal{B}_{\mathbb{F}_{\mathrm{cc}}}\left(\mathbb{R}^{n}\right)$, we have

$$
\pi(B) \in \mathcal{B}_{\Pi d_{H}}\left(\mathbb{R}^{n}\right)
$$

and for every $B \in \mathcal{B}_{\Pi d_{H}}\left(\mathbb{R}^{n}\right)$, we have

$$
\pi^{-1}(B) \in \mathcal{B}_{\mathbb{F}_{\mathrm{cc}}}\left(\mathbb{R}^{n}\right),
$$

where $\mathcal{B}_{\Pi d_{H}}\left(\mathbb{R}^{n}\right)$ is the set of Borel subsets for the topological space $\left(\mathrm{K}_{\mathrm{cc}}\left(\mathbb{R}^{n}\right)^{(0,1] \cap \mathbb{Q}}, \tau_{\Pi d_{H}}\right)$ and $\mathcal{B}_{\mathbb{F}_{\mathrm{cc}}}\left(\mathbb{R}^{n}\right)$ is that for $\left(\mathbb{F}_{\mathrm{cc}}\left(\mathbb{R}^{n}\right), \tau_{\mathbb{F}_{\mathrm{cc}}}\right)$.

Hereafter, when the fuzzy set $\widetilde{U}$ is the element of $\mathbb{F}_{\mathrm{cc}}\left(\mathbb{R}^{n}\right)$, it is called ( $n$-dimensional) fuzzy vectors in this paper. Especially it is called the fuzzy number when $n=1$. Fuzzy vectors and fuzzy numbers will be represented by lowercase letters with tilde like $\tilde{u}, \tilde{v}$, etc.

\section{Fuzzy Random Vectors as Vague Perception of Complex Random Phenomena}

In order to define fuzzy random vectors as vague but smart synthetic perception of complex random phenomena, several examples are investigated as follows:

Example 4.1. As a first example, suppose that a person feels the atmospheric temperature and he expresses linguistically his feeling like "about $5^{\circ} \mathrm{C}$, "about $10^{\circ} \mathrm{C}$, "about $15^{\circ} \mathrm{C}$, and so on.

The linguistic data in Example 4.1 has two outstanding features. One is fuzziness due to the intrinsic vagueness of language, and another is the randomness caused by the random fluctuation of the temperature. This means that the random variable, say $x_{0}$ defined on some probability space $(\Omega, \mathfrak{A}, P)$ for describing the temperature, is observed vaguely and expressed its value as linguistic vague data given by the fuzzy set like "about $5{ }^{\circ} \mathrm{C}$, "about $10{ }^{\circ} \mathrm{C}$,"about $15^{\circ} \mathrm{C}$ and so on. Consider here the subsets $\mathrm{A}_{1}, \mathrm{~A}_{2}, \cdots$ of the sample space $\Omega$ by

$A_{1}=\left\{\omega \mid\right.$ the value of $x_{0}(\omega)$ is expressed as "about $\left.5^{\circ} C^{\prime \prime}\right\}$

$\mathrm{A}_{2}=\left\{\omega \mid\right.$ the value of $x_{\mathrm{o}}(\omega)$ is expressed as "about $10^{\circ} \mathrm{C}$ " $\}$

$\mathrm{A}_{3}=\left\{\omega \mid\right.$ the value of $x_{\mathrm{o}}(\omega)$ is expressed as "about $15^{\circ} \mathrm{C}$ " $\}$

where $A_{1}, A_{2}, \cdots$ are assumed to the elements of $\sigma$-algebra $\mathfrak{A}$. Then, we can say that the generating point in each subset $\mathrm{A}_{i}(i=1,2, \cdots)$ may not be discriminated. This implies that the probabilistic information concerned with the random variable $x_{0}$ is decayed due to the vague perception, and this information decrease may be expressed by the mapping like $x_{\mathrm{o}}(\omega) \mapsto x^{*}(\omega)$, where $x^{*}$ is a $\mathfrak{A}^{*}$-measurable random vector with the sub $\sigma$-algebra $\mathfrak{A}^{*}$ of $\mathfrak{A}$ including each subset $\mathrm{A}_{i}(i=1,2, \cdots)$; and the remained information on $x_{\mathrm{o}}$ is for instance, the expected value of $x_{0}$. Therefore, it follows $E\left(x_{0}\right)=E\left(x^{*}\right)$, which implies that $x^{*}$ may be adequate to consider

$$
x^{*}(\omega)=E\left(x_{\mathrm{o}} \mid \mathfrak{A}^{*}\right),
$$

because of

$$
\int_{A} x^{*} d P=\int_{A} x d P \text { for every } A \in \mathfrak{A}^{*}
$$

Next, we consider a little more complex situation.

Example 4.2. Consider the situation that a person feels the temperature, humidity and the strength of wind simultaneously, and he expresses his feeling like "sultry", "comfortable", "humid", "muggy" and so on.

Premising that the temperature, humidity and strength of wind in Example 4.2 change randomly, the random vector

$$
\begin{aligned}
u_{0} & =(\text { temperature, humidity, strength of wind })^{\prime} \\
& =\left(u_{0,1}, u_{0,2}, u_{0,3}\right)^{\prime}
\end{aligned}
$$

is observed synthetically and expressed its value vaguely as a fuzzy set like "sultry", "comfortable", "humid" "muggy", etc. This means that we perceive the comfortableness synthetically and express it linguistically with the fuzzy set which includes the vague information on the realized value of the random vector $u_{0}$. In this example the subsets $\mathrm{A}_{1}, \mathrm{~A}_{2}, \cdots$ of the sample space $\Omega$ may be given by

$$
\begin{aligned}
& \mathrm{A}_{1}=\left\{\omega \mid \text { the value of } x_{0}=f\left(u_{0}(\omega)\right) \text { is felt as "sultry" }\right\} \\
& \mathrm{A}_{2}=\left\{\omega \mid \text { the value of } x_{0}=f\left(u_{0}(\omega)\right)\right. \text { is felt } \\
& \text { as "comfortable" }\} \\
& \mathrm{A}_{3}=\left\{\omega \mid \text { the value of } x_{0}=f\left(u_{0}(\omega)\right) \text { is felt as "humid" }\right\}
\end{aligned}
$$

where the function $x_{\mathrm{o}}=f\left(u_{\mathrm{o}}\right)$ describes some structure of the synthetic evaluation of the comfortableness, for instance, 
it may be given by $x_{0}=f\left(u_{0}\right)=a \cdot u_{0,1}+b \cdot u_{0,2}+c \cdot u_{0,3}$, where $a, b, c$ are some constants. Furthermore, as same as in Example 4.1, assuming $A_{1}, A_{2}, \cdots$ are the elements of $\mathfrak{A}$, we can say again that the generating point in each subset $\mathrm{A}_{i}(i=1,2, \cdots)$ may not be discriminated. This implies that the probabilistic information concerned with the random vector $x_{0}$ is decayed due to the vague perception, and its information decrease may be expressed by the mapping like

$$
x_{0}(\omega)=f\left(u_{0}(\omega)\right) \mapsto x^{*}(\omega),
$$

where $x^{*}$ is a $\mathfrak{A}^{*}$-measurable random vector with the sub $\sigma$-algebra $\mathfrak{A}^{*}$ of $\mathfrak{A}$ including each subset $\mathrm{A}_{i}(i=1,2, \cdots)$; and assuming that the remained information on $x_{0}$ is the expected value of $x_{0}$, we can get (17).

Finally, we consider more complex example.

Example 4.3. Consider the situation of evaluating the economic condition for forecasting the business activities. It is needless to say that the economic condition is evaluated from various economic indices such as stock prices, exchange rates of money, the unemployment rate, etc. However, each economic index shows only one aspect of the highly complex economic system and it is often observed that at first glance, some of the indices seem to show the contradictory values each other. Therefore, deliberating the whole aspect of the economic system indicated by various indices, we have to evaluate the economic state and have to express it like "active", "not so active", "good", "bad" and so on. Furthermore, it should be also taken into consideration that the economic condition fluctuates randomly due to the unpredictable changes of economic environments.

Assuming that the economic indices $u_{0,1}, u_{0,2}, \cdots, u_{0, n}$ in Example 4.3 change randomly, the random vector

$$
u_{0}=\left(u_{0,1}, u_{0,2}, \cdots, u_{0, n}\right)^{\prime}
$$

defined on some probability space $(\Omega, \mathfrak{A}, P)$ is linguistically and synthetically perceived as the vague random data given by the fuzzy set like "good", "bad", "neutral" and "not so bad", etc. This means that we can perceive the economic state vaguely but concisely as a fuzzy set which includes the imperfect information on the realized value of the random vector $u_{0} \in \mathbb{R}^{n}$ (i.e. the collection of some economic indices). In this example, the subsets $A_{1}, A_{2}, \cdots$ of the sample space $\Omega$ may be given by

$$
\begin{aligned}
& \mathrm{A}_{1}=\left\{\omega \mid \text { the value of } x_{0}=f\left(u_{0}(\omega)\right)\right. \text { is expressed } \\
& \text { as "good" }\} \\
& \mathrm{A}_{2}=\left\{\omega \mid \text { the value of } x_{0}=f\left(u_{0}(\omega)\right)\right. \text { is expressed } \\
& \text { as "bad" }\} \\
& \mathrm{A}_{3}=\left\{\omega \mid \text { the value of } x_{0}=f\left(u_{0}(\omega)\right)\right. \text { is expressed } \\
& \text { as "neutral" }\}
\end{aligned}
$$

where the function $x_{0}=f\left(u_{0}(\omega)\right) \in \mathbb{R}$ describes the structure of the synthetic perception, some part of which may be known but most part of which may be unknown. Furthermore, assuming that $A_{1}, A_{2}, \cdots$ are the elements of $\mathfrak{A}$, we can say that the generating point in each subset $\mathrm{A}_{i}(i=$ $1,2, \cdots)$ may not be discriminated as same as in Examples 4.1 and 4.2. This implies that the probabilistic information concerned with the random vector $x_{\mathrm{o}}$ in this example is also decayed due to the vague perception, and this information decrease may be expressed by the mapping (18) and assuming that the remained information on $x_{0}$ is its expected value, we can get (17).

According to the examples mentioned above, it may be reasonable to consider that the fuzzy random variables or more generally fuzzy random vectors(FRVCs for short) are defined as a some kind of $\mathfrak{A}^{*}-\mathcal{B}_{\mathbb{F}_{\mathrm{cc}}}\left(\mathbb{R}^{n}\right)$-measurable fuzzy functions as follows:

Definition 4.1. A FRVC $\tilde{x}$ obtained as the vague but synthetic perception of an ordinary random vector $u_{\mathrm{o}} \in \mathbb{R}^{n}$ is defined by the $\mathfrak{A}^{*}-\mathcal{B}_{\mathbb{F}_{\mathrm{cc}}}\left(\mathbb{R}^{n}\right)$-measurable fuzzy function as follows:

$$
\tilde{x}(\omega)=\left(\mathbb{R}^{n},\left\{\tilde{x}_{\alpha}(\omega) \mid \alpha \in I\right\}, s_{\tilde{x}}\right)
$$

where $s_{\tilde{x}}$ is the predicate associated with the statement such as

$$
s_{\tilde{x}}(x, \omega)=\left\{x \text { coincides with } x^{*}(\omega)=E\left(f\left(u_{0}\right) \mid \mathfrak{A}^{*}\right)\right\} .
$$

The dimension $m$ of $x^{*}$ may be much less than the dimension $n$ of $u_{0}$.

Remark: Assume here that the each element of the set representation $\left\{\tilde{x}_{\alpha} \mid \alpha \in(0,1)\right\}$ of a FRVC $\tilde{x}$ is $\mathfrak{A}^{*}-\mathcal{B}_{d_{H}}\left(\mathbb{R}^{n}\right)$ measurable. Then, it can be shown that

$$
\bigcap_{\beta \in(0, \alpha) \cap \mathbb{Q}} \tilde{x}_{\beta}=\operatorname{Pr}_{\alpha} \circ \pi \circ \tilde{x}
$$

is also $\mathfrak{A}^{*}-\mathcal{B}_{d_{H}}\left(\mathbb{R}^{n}\right)$-measurable. Let $\alpha_{1}, \alpha_{2}, \cdots \alpha_{r}$ be in $(0,1] \cap \mathbb{Q}$ and $G_{1}, G_{2}, \cdots, G_{r} \in \tau_{d_{H}}$. Then, we have

$$
(\pi \circ \tilde{x})^{-1}\left(\bigcap_{i=1}^{r} \operatorname{Pr}_{\alpha_{i}}^{-1}\left(G_{i}\right)\right)=\bigcap_{i=1}^{r}\left(\bigcap_{\beta \in\left(0, \alpha_{i}\right) \cap \mathbb{Q}} \tilde{x}_{\beta}\right)^{-1}\left(G_{i}\right) \in \mathfrak{A}^{*} .
$$

The set $\mathbb{B}$ defined by

$$
\begin{array}{r}
\mathbb{B}=\left\{\bigcap_{i=1}^{r} \operatorname{Pr}_{\alpha_{i}}^{-1}\left(G_{i}\right) \mid r \in \mathbb{N}, G_{1}, G_{2}, \cdots, G_{r} \in \tau_{d_{H}},\right. \\
\left.\alpha_{1}, \cdots, \alpha_{r} \in(0,1) \cap \mathbb{Q}\right\}
\end{array}
$$

constitute a basis of the product topology $\tau_{\Pi d_{H}}$, and $\left(\left(\mathrm{K}_{\mathrm{cp}}\left(\mathbb{R}^{n}\right)\right)^{(0,1] \cap \mathbb{Q}}, \tau_{\Pi d_{H}}\right)$ is a Polish space. Therefore, each $H \in \tau_{\Pi d_{H}}$ can be written as a union of a sequence $\left\{H_{i}, i \in \mathbb{N}\right\}$ in $\mathbb{B}$. Hence, it follows

$$
(\pi \circ \tilde{x})^{-1}(H)=\bigcup_{i=1}^{\infty}(\pi \circ \tilde{x})^{-1}\left(H_{i}\right) \in \mathfrak{A}^{*} .
$$

Then, it can be shown that the FRVCs previously proposed by the author[1] are those defined in Definition4.1. 
Proposition 4.1. Let $\tilde{x}=\left(\mathbb{R}^{n},\left\{\tilde{x}_{\alpha} \mid \alpha \in I\right\}, s_{\tilde{x}}\right)$ be a FRVC. Then, the strong cut $L_{\alpha} \tilde{x}(\omega)$ is an $\mathfrak{A}^{*}-\mathcal{B}_{d_{H}}\left(\mathbb{R}^{n}\right)$-measurable correspondence for $\alpha \in[0,1)$, and the level set $L \bar{\alpha} \tilde{x}(\omega)$ is a $\mathfrak{L}^{*}-\mathcal{B}_{d_{H}}\left(\mathbb{R}^{n}\right)$-measurable correspondence for $\alpha \in(0,1]$.

In order to define the expectation of a FRVC, the integrability of FRVCs is defined by using that of random correspondences:

Definition 4.2. A FRVC $\tilde{x}(\omega)=\left(\mathbb{R}^{n},\left\{\tilde{x}_{\alpha} \mid \alpha \in I\right\}, s_{\tilde{x}}\right)$ is called integrable, if the random correspondence $\tilde{x}_{1}(\omega)$ is integrable, i.e., $S_{\tilde{x}_{1}}\left(\mathfrak{A}^{*}\right)$ defined by

$$
\begin{aligned}
S_{\tilde{x}_{1}}\left(\mathfrak{A}^{*}\right)= & \left\{x \mid x \in \mathbb{L}\left[\Omega, \mathfrak{A}^{*}, P ; \mathbb{R}^{n}\right],\right. \\
& \left.x(\omega) \in \tilde{x}_{1}(\omega) \text { a.s. on }\left(\Omega, \mathfrak{A}^{*}, P\right)\right\}
\end{aligned}
$$

is nonempty, where $\mathbb{L}\left[\Omega, \mathfrak{A}^{*}, P ; \mathbb{R}^{n}\right]$ is the Banach space of $\mathfrak{A}^{*}$-measurable mapping $g: \Omega \rightarrow \mathbb{R}^{n}$ with the norm $\|g\|=$ $\int_{\Omega}\|g(\omega)\| d P$.

\section{Expectation of FRVCs}

In order to explore the reasonable defining method for the expectation of a FRVC $\tilde{x}$, consider first the statement given by

$$
s_{\mathcal{E}[\tilde{x}]}(x)=\left\{x \text { coincides with the expectation of } x^{*}\right\},
$$

where $x^{*}$ is the $\mathfrak{A}^{*}$-measurable random vector satisfying (20). Then, it may be considered that above statement is given as a composite statement such as

$$
\begin{array}{r}
s_{\mathcal{E}[\tilde{x}]}(x)=\bigvee_{\xi^{*} \in \mathbb{L}\left[\Omega, \mathfrak{A} *, P ; \mathbb{R}^{n}\right]}\left\{\left(x=E\left\{\xi^{*}\right\}\right)\right. \\
\left.\wedge\left(\xi^{*}=x^{*} \text { a.s. on }\left(\Omega, \mathfrak{A}^{*}, P\right)\right)\right\},
\end{array}
$$

where $\xi^{*}=E\left(\xi \mid \mathfrak{A}^{*}\right)$ and $\xi$ is the possible original random vector. Furthermore, the statement " $\xi^{*}=x^{*}$ a.s. on $\left(\Omega, \mathfrak{A}^{*}, P\right)^{\prime}$ in $(24)$ is given by

$$
\begin{aligned}
\left(\xi^{*}=x^{*} \text { a.s. on }\left(\Omega, \mathfrak{A}^{*}, P\right)\right) & =\bigwedge_{\omega \in \Omega \backslash N_{0}}\left(\xi^{*}(\omega)=x^{*}(\omega)\right) \\
= & \bigwedge_{\omega \in \Omega \backslash \mathrm{N}_{0}} s_{\tilde{x}}\left(\xi^{*}, \omega\right)
\end{aligned}
$$

where $\mathrm{N}_{0}$ is the null set, i.e., $P\left(\mathrm{~N}_{0}\right)=0$ with $\mathrm{N}_{0} \in \mathfrak{A}^{*}$ and $s_{\tilde{x}}\left(\xi^{*}, \omega\right)$ is the statement given by (20). Then, (24) is rewritten by

$S_{\mathcal{E}[\tilde{x}]}(x)$

$$
=\bigvee_{\xi^{*} \in \mathbb{L}\left[\Omega, \mathfrak{A}^{*}, P ; \mathbb{R}^{n}\right]}\left\{\left(x=E\left\{\xi^{*}\right\}\right) \wedge\left(\bigwedge_{\omega \in \Omega \backslash \mathrm{N}_{0}} s_{\tilde{x}}\left(\xi^{*}, \omega\right)\right)\right\} .
$$

Therefore, applying the concept of the extension principle for fuzzy sets proposed by Zadeh[8], it may be considered that the truth value of $s \varepsilon[\tilde{x}](x)$ is given by

$$
\begin{aligned}
& t\left(s_{\mathcal{E}[\tilde{x}]}(x)\right) \\
& =\sup _{\xi^{*} \in \mathbb{L}\left[\Omega, \mathfrak{A}^{*}, P ; \mathbb{R}^{n}\right]}\left\{\operatorname{essinf}_{\omega \in \Omega} t\left(s_{\tilde{x}}\left(\xi^{*}, \omega\right)\right) \mid x=E\left\{\xi^{*}\right\}\right\},
\end{aligned}
$$

where essinf $\operatorname{s\in \Omega }_{\omega \in \Omega} t\left(s_{\tilde{x}}\left(\xi^{*}, \omega\right)\right)$ is given by the supremum of $a$ satisfying

$$
t\left(s_{\tilde{x}}\left(\xi^{*}, \omega\right)\right)=(\tilde{x}(\omega))\left(\xi^{*}\right) \geq a \text { a.s. on }\left(\Omega, \mathfrak{A}^{*}, P\right)
$$

Then, we can confirm the following property holds:

Proposition 5.1. Let $\tilde{x}$ be a FRVC, and $\left\{\tilde{x}_{\alpha} \mid \alpha \in I\right\}$ be its set representation. Then,

$$
\begin{aligned}
\left\{x \mid t\left(s_{\mathcal{E}[\tilde{x}]}(x)\right)>\alpha\right\} \subseteq E\left[\tilde{x}_{\alpha}\right] & \\
& \subseteq\left\{x \mid t\left(s_{\varepsilon[\tilde{x}]}(x)\right) \geq \alpha\right\} \quad \text { for } \forall \alpha \in I
\end{aligned}
$$

where $E\left[\tilde{x}_{\alpha}\right]$ is the Aumann Integral of $\tilde{x}$, i.e.,

$$
E\left[\tilde{x}_{\alpha}\right]=\int_{\Omega}^{\left\{\mathfrak{A}^{*}\right\}} \tilde{x}_{\alpha} d P=\left\{\int_{\Omega} x d P ; x \in S_{\tilde{x}_{\alpha}}\left(\mathfrak{A}^{*}\right)\right\}
$$

with

$$
\begin{aligned}
S_{\tilde{x}_{\alpha}}\left(\mathfrak{A}^{*}\right)=\mathrm{cl} . & \left\{\xi^{*} \mid \xi^{*} \in \mathbb{L}\left[\Omega, \mathfrak{A}^{*}, P ; \mathbb{R}^{n}\right],\right. \\
& \left.\xi^{*}(\omega) \in \tilde{x}_{\alpha}(\omega) \text { a.s. on }\left(\Omega, \mathfrak{A}^{*}, P\right)\right\} .
\end{aligned}
$$

proof: It is clear from (8a) that

$$
L_{\alpha} \tilde{x} \subseteq \tilde{x}_{\alpha} \subseteq L_{\bar{\alpha}} \tilde{x} \text { for each } \alpha \in I .
$$

Then, we have that

$$
S_{L_{\alpha} \tilde{x}}^{1}\left(\mathfrak{A}^{*}\right) \subseteq S_{\tilde{x}_{\alpha}}^{1}\left(\mathfrak{A}^{*}\right) \subseteq S_{L_{\alpha^{*}} \tilde{x}}^{1}\left(\mathfrak{A}^{*}\right),
$$

which follows

$$
\int_{\Omega}^{\left\{\mathfrak{a}^{*}\right\}} L_{\alpha} \tilde{x} d P \subseteq \int_{\Omega}^{\left\{\mathfrak{A}^{*}\right\}} \tilde{x}_{\alpha} d P \subseteq \int_{\Omega}^{\left\{\mathfrak{A}^{*}\right\}} L_{\bar{\alpha}} \tilde{x} d P .
$$

(i) If there exists a vector $\zeta$ such that

$$
\begin{aligned}
& \zeta \in\left\{x \mid t\left(s_{\mathcal{E}[\tilde{x}]}(x)\right)>\alpha\right\} \\
&=\left\{x \mid \sup _{\xi^{*} \in \mathbb{L}\left[\Omega, \mathfrak{A}^{*}, P ; \mathbb{R}^{n}\right]}\left\{\left(\underset{\omega \in \Omega}{\operatorname{essinf}}(\tilde{x}(\omega))\left(\xi^{*}(\omega)\right)\right)\right.\right. \\
&\left.\left.\mid x=E\left(\xi^{*}\right)\right\}>\alpha\right\},
\end{aligned}
$$

there is some element $\xi^{*}$ of $\mathbb{L}\left[\Omega, \mathfrak{A}^{*}, P ; \mathbb{R}^{n}\right]$ satisfying $E\left(\xi^{*}\right)=\zeta$ and

$$
\xi^{*}(\omega) \in L_{\alpha} \tilde{x}(\omega) \text { a.s. on }\left(\Omega, \mathfrak{A}^{*}, P\right)
$$


It is clear that $\xi^{*}$ in (36) is the element of $S_{L_{\alpha} \tilde{x}}\left(\mathfrak{A}^{*}\right)$ and hence we have

$$
\zeta=E\left(\xi^{*}\right)=\int_{\Omega} \xi^{*} d P \in \int_{\Omega}^{\left\{\mathfrak{A}^{*}\right\}} L_{\alpha} \tilde{x} d P,
$$

or equivalently we can conclude that

$$
\left\{x \mid t\left(s_{\mathcal{E}[\tilde{x}]}(x)\right)>\alpha\right\} \subseteq \int_{\Omega}^{\left\{\mathfrak{X}^{*}\right\}} L_{\alpha} \tilde{x} d P=E\left[L_{\alpha} \tilde{x}\right] .
$$

(ii) If there exists $\zeta$ such that

$$
\zeta \in \int_{\Omega}^{\left\{\mathfrak{A}^{*}\right\}} L_{\bar{\alpha}} \tilde{x} d P=\left\{x \mid x=\int_{\Omega} \xi^{*} d P, \xi^{*} \in S_{L_{\tilde{\alpha}} \tilde{x}}^{1}\left(\mathfrak{L}^{*}\right)\right\},
$$

there is some element $\xi^{*}$ of $\mathbb{L}\left[\Omega, \mathfrak{A}{ }^{*}, P ; \mathbb{R}^{n}\right]$ satisfying $\zeta=$ $\int_{\Omega} \xi^{*} d P$ and

$$
(\tilde{x}(\omega))\left(\xi^{*}(\omega)\right) \geq \alpha \quad \text { a.s. on }\left(\Omega, \mathfrak{A}^{*}, P\right) .
$$

(40) implies that there exists $\xi^{*} \in \mathbb{L}\left[\Omega, \mathfrak{A}^{*}, P ; \mathbb{R}^{n}\right]$ satisfying $\zeta=E\left(\xi^{*}\right)$ and

$$
\underset{\omega \in \Omega}{\operatorname{essinf}}(\tilde{x}(\omega))\left(\xi^{*}(\omega)\right) \geq \alpha,
$$

which means that

$$
\zeta \in\left\{x \mid t\left(s_{\mathcal{E}[\tilde{x}]}(x)\right) \geq \alpha\right\},
$$

or equivalently

$$
\int_{\Omega}^{\left\{\mathfrak{A}^{*}\right\}} L_{\bar{\alpha}} \tilde{x} d P=E\left[L_{\bar{\alpha}} \tilde{x}\right] \subseteq\left\{x \mid t\left(s_{\mathcal{E}[\tilde{x}]}(x)\right) \geq \alpha\right\} .
$$

(iii) From (34), (i) and (ii), we have

$$
\begin{aligned}
&\left\{x \mid t\left(s_{\mathcal{E}[\tilde{x}]}(x)\right)>\alpha\right\} \subseteq E\left[L_{\alpha} \tilde{x}\right] \subseteq E\left[\tilde{x}_{\alpha}\right] \\
& \subseteq E\left[L_{\bar{\alpha}} \tilde{x}\right] \subseteq\left\{x \mid t\left(s_{\mathcal{E}[\tilde{x}]}(x)\right) \geq \alpha\right\}
\end{aligned}
$$

for each $\alpha \in I$.

Q.E.D.

Then, the following definition of the expectation of a FRVC $\tilde{x}$ may be reasonable:

Definition 5.1. Let $\tilde{x}=\left(\mathbb{R}^{n},\left\{\tilde{x}_{\alpha} \mid \alpha \in I\right\}, s_{\tilde{x}}\right)$ be an integrable FRVC. Then, the expectation of $\tilde{x}$ is defined by

$$
\mathcal{E}[\tilde{x}]=\left(\mathbb{R}^{n},\left\{E\left[\tilde{x}_{\alpha}\right] \mid \alpha \in I\right\}, s_{\varepsilon[\tilde{x}]}\right),
$$

where $s_{\mathcal{E}[x]}$ is the predicate associated with the statement given by (23); and $\left\{E\left[\tilde{x}_{\alpha}\right] \mid \alpha \in I\right\}$ is the set representation of $\mathcal{E}[\tilde{x}]$ given through (30).

It is clear from (29) that

$$
L_{\alpha} \varepsilon[\tilde{x}] \subseteq E\left[\tilde{x}_{\alpha}\right] \subseteq L_{\bar{\alpha}} \varepsilon[\tilde{x}] \quad \text { for } \forall \alpha \in I
$$

with

$$
\begin{aligned}
& L_{\alpha} \varepsilon[\tilde{x}]=\{x \mid(\varepsilon[\tilde{x}])(x)>\alpha\}, \\
& L_{\bar{\alpha}} \varepsilon[\tilde{x}]=\{x \mid(\mathcal{E}[\tilde{x}])(x) \geq \alpha\}
\end{aligned}
$$

and

$$
(\mathcal{E}[\tilde{x}])(x)=t\left(s_{\mathcal{E}[\tilde{x}]}(x)\right),
$$

which means that $\left\{E\left[\tilde{x}_{\alpha}\right] \mid \alpha \in I\right\}$ surely satisfies the condition imposed on set representations of fuzzy sets given by (8a).

The following corollary obtained immediately from (44) in the proof of Proposition 5.1.

Corollary 5.1. Let $\tilde{x}$ be an integrable FRVC and $\left\{\tilde{x}_{\alpha} \mid \alpha \in I\right\}$ be its set representation. Then we have

$$
L_{\alpha} \varepsilon[\tilde{x}] \subseteq E\left[L_{\alpha} \tilde{x}\right] \subseteq \mathcal{E}\left[\tilde{x}_{\alpha}\right] \subseteq E\left[L_{\bar{\alpha}} \tilde{x}\right] \subseteq L_{\bar{\alpha}} \mathcal{E}[\tilde{x}] .
$$

\section{References}

[1] T. Fukuda. On a class of fuzzy random vectors(in Japanese). Journal of Japan Society for Fuzzy Theory and Systems, 10(3):499-505, 1998.

[2] V. Krätschmer. A unified approach to fuzzy random variables. Fuzzy Sets and Systems, 123:1-9, 2001.

[3] H. Kwakernaak. Fuzzy random variables-I: Definitions and theorems. Information Sciences, 15:1-29, 1978.

[4] R. E. Bellman and M. Giertz. On the analytic formalism of the theory of fuzzy sets. Information Sciences, 5:149-156, 1973 .

[5] M. Mukaidono. Fuzzy Logic(in Japanese). NikkanKougyo Shinbun, Tokyo, Japan, 1993.

[6] R. Kruse and K. D. Meyer. Statistics with Vague Data. D. Reidel Publishing Company, Dordecht, 1987.

[7] R. Kruse, J. Gebhardt, and F. Klawonn. Foundations of Fuzzy Systems. John Wiley \& Sons, Chichester, 1994.

[8] L. A. Zadeh. The concept of a linguistic variables and its application in approximate reasoning, parts 1,2 and 3. Information Sciences, 8 and 9:199-249,301-357,4380, respectively, 1975,76 . 